\title{
Test-retest Reliability of Ocular Vestibular Myogenic Potential in Healthy Pilots G Meng ${ }^{1}$, C Shan ${ }^{1}$, L Han ${ }^{1}$, SJ Xie ${ }^{2}$
}

\begin{abstract}
Background: Vestibular function is essential to pilots. But for now there are little method to evaluate otolith function in pilots. Quite recently the ocular vestibular evoked myogenic potential (oVEMP) test has been introduced to evaluate the utricular and the superior vestibular nerve function. The test-retest reliability of oVEMP by air conducted sound is crucial and indispensable for its application in pilots. Hence, the purpose of the present study is to assess the reliability of oVEMP by air conducted sound in pilots.

Methods: Twenty Chinese Air Force active-duty fighter pilots (10 females and 10 males with a mean age of 32 years) were recruited as study participants. Each subject underwent oVEMP test with an interval of one week. Acoustic stimuli as $97 \mathrm{~dB}$ nHL short tone bursts $(\mathrm{STB})[500 \mathrm{~Hz}$, rise/fall time $=$ $1 \mathrm{~ms}$, plateau time $=2 \mathrm{~ms}$ ] with rarefaction polarity were delivered through the insert earphones to evoked the oVEMP. The responses to 100 stimuli were averaged and the latencies of the $\mathrm{nI}$ and $\mathrm{pI}$ peak, peak to peak amplitude were determined, as well as the asymmetry ratio between the peak to peak amplitudes of oVEMP. Agreement between the parameters of oVEMP on the two different test days with an interval of one week was demonstrated by intra class correlation tests.
\end{abstract}

Results: Among the parameters of oVEMP test, reliability was excellent for n10 latency, peak-to-peak amplitude and AR for peak-to-peak amplitude whereas reliability was fair-to-good for pI latency.

Conclusions: Good to excellent test-retest reliability has been established for overall parameters of oVEMP in 20 healthy pilots, with the latency of n10 more reliable than the latency of p15. It implies that oVEMP test is reliable in the vestibular evaluation of pilots.

Keywords: Military personnel, vestibular-evoked myogenic potential (VEMP), utricule reliability

From: ${ }^{1}$ Nautical and Aviation Medical Centre, Navy General Hospital, PR China and ${ }^{2}$ Vestibular function laboratory, Institute of Aviation Medicine, Air Force, PR China.

Correspondence: Dr G Meng, No 6 Fucheng Road, Haidian District, Beijing, PR China

Zipcode: 100048, E-mail: 377379056@qq.com 


\section{oVEMP Reliability Pilots}

\section{INTRODUCTION}

Normal and effective vestibular system function is especially an absolute precondition for high performance fighter pilots. Not only is it necessary to exclude inner ear diseases such as Meniere's disease, benign paroxysmal positional vertigo, vestibular neuritis, vestibular schwannoma, labyrinthitis, vestibular migraine etc, but it is also important for the pilot to be able to produce symmetrical and sufficiently balanced responses in order to successfully fight against spatial disorientation. Consequently comprehensive vestibular function examination is considered as an important and responsible task in the selection of pilot candidates and aeromedical evaluation of pilots with oto-neurological diseases.

Vestibular end organs comprise three semicircular canals and two otolithic organs, ie saccule and utricle. Clinically, semicircular canals are assessed by caloric test with videonystagmography or the head impulse test (1), whereas safe simple tests of otolith function are not common. Recently, two important tests of otolith function using the vestibular-evoked myogenic potential (VEMP) test have been reported. By means of air-conducted sound (ACS) stimulation, VEMP can be elicited from tonically contracted cervical muscles, the so-called cervical VEMP (cVEMP), which has been shown to originate from the saccule (2). Recent investigations revealed that VEMP could also be recorded from extraocular muscles, the so-called ocular VEMP (oVEMP), which was elicited by ACS and referred to the utricular function via a crossed vestibulo-ocular reflex [VOR] (3-5). Unlike the cVEMP test, which assesses ipsilateral saccular fuction (2), the oVEMP test has been validated for evaluating contralateral utricular function (3-5). The first biphasic potential of cVEMP has a positive peak (p) with a mean latency of 13 milliseconds (ms), followed by a negative peak (n) with a mean latency of $23 \mathrm{~ms}$, denominated p13-n23 (2). While the first biphasic potential of oVEMP has a negative peak (n) with a mean latency of 10 milliseconds (ms), followed by a peak positive (p) with a mean latency of $15 \mathrm{~ms}$, denominated n10-p15 


\section{Meng et al}

(3-5). For now oVEMP test has been introduced to evaluate the utricular-ocular reflex and the superior vestibular nerve function in the clinical settings.

However, so far as we know, oVEMP has not been applied in the medical selection and physiological assessment of fighter pilots due to lack the evaluation of its reliability in healthy pilots. Hence, the aim of the present paper is to assess the reliability of oVEMP parameters in Chinese Air Force fighter pilots so as to prompt the application of oVEMP in pilots.

\section{SUBJECTS and METHODS}

Twenty healthy Chinese Air Force active-duty jet fighter pilots (10 females and 10 males, with a mean age of 32 and a range of 23-48 years as well as a mean flight time of $1222.8 \pm$ 450.9 hours) who served as study participants. All participants had to be mentally and physically fit according to the standards of Medical Fitness for Chinese Air Force aircrew.

None of them had any history of inner ear diseases or dizziness, hearing loss or tinnitus. None of them also had any history of eye diseases or eye movement dysfunction. The hearing level was normal in all participants according to ISO 7029 (6). Horizontal head impulse, headshaking, vibration-induced nystagmus test with Frenzel goggles, subjective visual vertical tests, and caloric tests were also within normal limits in all participants. Every subject underwent two oVEMP test with a time interval of one week. The study protocol was approved in advance by the Chinese Air Force Institute of Aviation Medicine Institutional Review Board. Each subject provided written informed consent before participation after all procedures had been fully explained. 
oVEMP Reliability Pilots

\section{Equipment}

The subjects were in a sitting position. Surface potentials, predominantly electromyographic (EMG) activities, were recorded (Smart EP 3.90, Intelligent Hearing Systems, Miami, Fla, USA). Two active electrodes were placed bilaterally around $1 \mathrm{~cm}$ below the center of lower eyelid. The other two reference electrodes were respectively positioned about $1-2 \mathrm{~cm}$ below the active ones, and one ground electrode was placed on the sternum (7). The electrode impedance was kept under $5 \mathrm{k} \Omega$. During recording, the subjects were instructed to look upward at a small fixed target more than $2 \mathrm{~m}$ away from the eyes, with a vertical visual angle of approximately $30-35^{\circ}$ above horizontal.

\section{Procedure}

Acoustic stimuli as $97 \mathrm{~dB}$ nHL short tone bursts (STB) $[500 \mathrm{~Hz}$, rise/fall time $=1 \mathrm{~ms}$, plateau time $=2 \mathrm{~ms}]$ with rarefaction polarity were delivered through the insert earphones. Stimuli were presented for 100 repetitions at a rate of about $5 \mathrm{~Hz}$. EMG was amplified and bandpass filtered $[1 \mathrm{~Hz}-1 \mathrm{k} \mathrm{Hz}](8)$ then sampled at $10 \mathrm{k} \mathrm{Hz}$ from $20 \mathrm{~ms}$ before to $30 \mathrm{~ms}$ following stimulus onset and averaged. Bilateral oVEMPs were recorded simultaneously using binaural acoustic stimulation according to Wang et al (9).

\section{oVEMP response parameters}

The oVEMP waveform was comprised of a initial negative peak with a mean latency of around $10 \mathrm{~ms}$ (n10), followed by a initial positive peak with a mean latency of around $15 \mathrm{~ms}$ (p15). Consecutive runs were performed to confirm the reproducibility of peak n10 and p15, which were interpreted by two independent observers and then oVEMPs were deemed to be present. Conversely, oVEMPs were absent when the biphasic waveform was not reproducible. The latencies of peak $\mathrm{n} 10$ and p15, n10 - p15 amplitude and asymmetry ratio (AR) were 


\section{Meng et al}

measured. The latter $(\%)$ was defined as the difference of the amplitude n10 - p15 on each ear divided by the sum of amplitude n10 - p15 of both ears, that is ((larger amplitude smaller amplitude)/ (larger amplitude + smaller amplitude) $) \times 100$.

\section{Statistical Analysis}

Test-retest reliability was assessed with the intraclass correlation coefficient (ICC), calculated using a two-way random effects, absolute agreement model with a statistical significance level of $p<0.05(10)$. In the present study, the ICC is the ratio of the variance of a VEMP parameter due to subject differences divided by the sum of variances due to subject differences and measurement errors. For a oVEMP test to be clinically useful, variance over repeated measures should, at minimum, reflect physiological differences between subjects more than changes in the measurement "noise" between testing sessions. That is, ICC should be $\geqslant 0.5$. Therefore, for each ICC value we determined its significance based on this criterion using an F-test with the number of subjects, two observations, and a null-hypothesis value of $\mathrm{ICC}_{0}=0.5$ at an $\alpha$ level of 0.05 . In general, the number of observations for monaural parameters $(\sim 40)$ was sufficient to detect ICC values of $\geqslant 0.8$ as significantly different than $\mathrm{ICC}_{0}=0.5(p<0.05)$ with a power of 0.8 . Similar to previous studies that examined VEMP test-retest reliability, we classified an ICC value of 1.00 as perfect reliability, ICC $\geqslant 0.75$ as excellent reliability, $0.40 \leqslant$ ICC $<0.75$ as fair-to-good reliability and ICC $<0.40$ as poor reliability $(10-12)$. Intraclass correlation coefficient analysis was performed using SPSS 17.0 (Chicago, IL) and standard deviation analysis was performed using SPSS for Windows version 15. 


\section{RESULTS}

All 20 subjects (40 ears) had clear oVEMPs elicited by ACS in two test sessions. Figure 1 shows oVEMP waveforms obtained form both the first and second testing sessions in a fighter pilot. The mean values and standard deviations of the oVEMP n10 and p15 latencies, $\mathrm{n} 10$ - p15 interval, peak-to-peak n10 - p15 amplitude, and asymmetry ratio for the first and second testing sessions are showed in (Table 1).

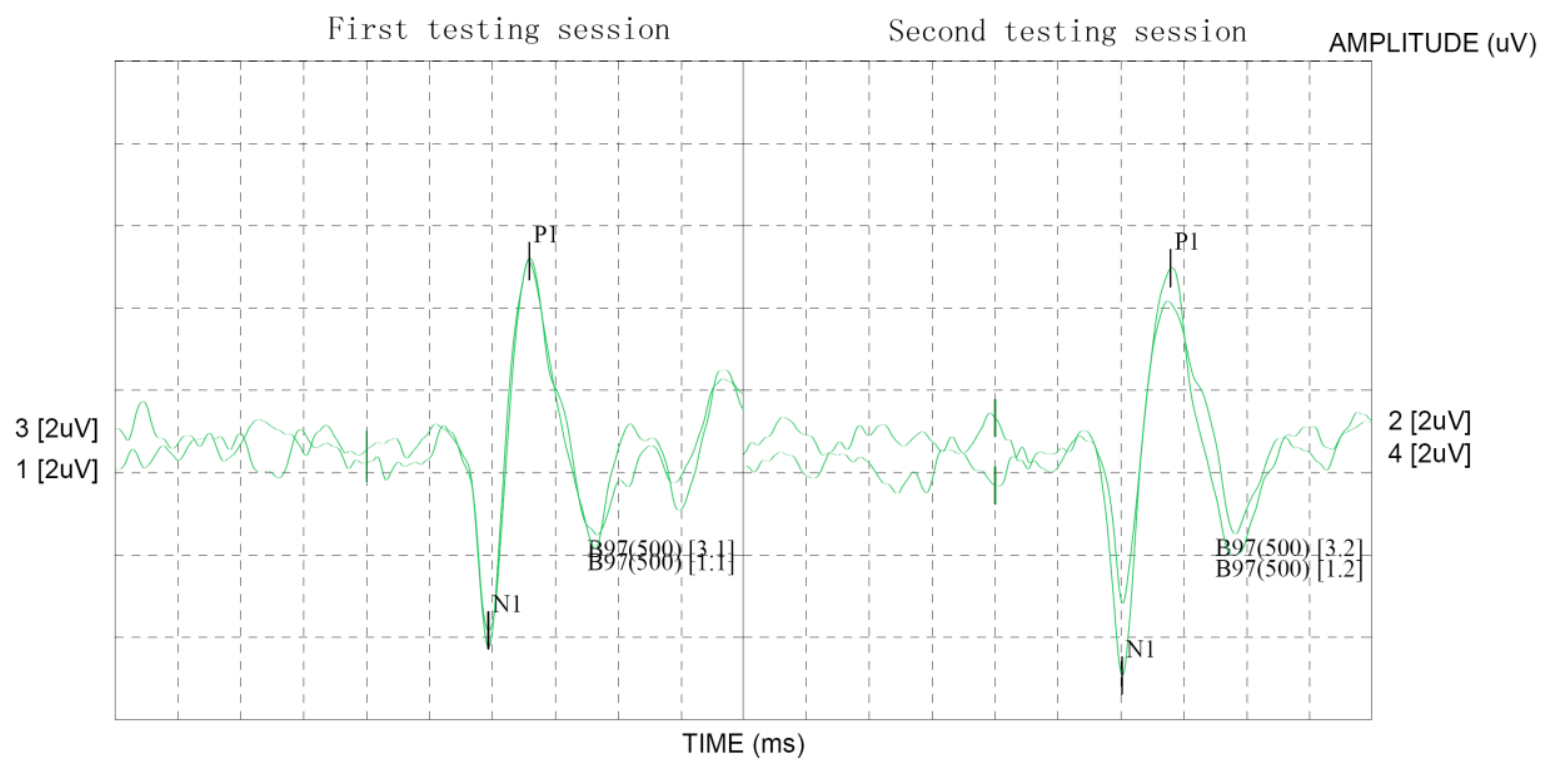

Fig: 1. Ocular VEMP traces from a pilot in the first and second testing sessions.

Table. 1: Mean and standard deviations of oVEMP test parameters from the first and second testing sessions (40 ears)

\begin{tabular}{lcc}
\hline & First session & Second session \\
\hline N 10 latency $(\mathrm{ms})$ & $10.38 \pm 0.66$ & $10.34 \pm 0.73$ \\
P15 latency $(\mathrm{ms})$ & $15.31 \pm 1.18$ & $15.07 \pm 0.88$ \\
Peak to peak amplitude $(\mu \mathrm{V})$ & $6.96 \pm 3.85$ & $7.02+3.92$ \\
AR for peak-to-peak amplitude $(\%)$ & $13.09 \pm 9.18$ & $13.40 \pm 9.43$ \\
\hline
\end{tabular}

Table 2 showed the ICC values for oVEMP test-retest reliability. Intraclass correlation coefficient values indicting excellent reliability are marked with bold font. Among the parameters of oVEMP test, reliability was excellent for n10 latency, peak-to-peak 
amplitude and AR for peak-to-peak amplitude whereas reliability was fair-to-good for p15 latency.

Table. 2: Intraclass correlation coefficient values for oVEMP test-retest reliability

\section{ICC values}

\begin{tabular}{ll}
\hline N 10 latency & $0.92 *(0.62,0.96)$ \\
P15 latency & $0.74 *(0.38,0.90)$ \\
Peak to peak amplitude & $0.85 *(0.53,0.94)$ \\
AR for peak-to-peak amplitude & $0.93 *(0.41,0.98)$ \\
\hline
\end{tabular}

Note: Intraclass correlation coefficient values denoting excellent reliability are marked in bold and the corresponding confidence intervals are shown in parentheses. Asterisks denote excellent reliability values that furthermore were statistically significant at the $5 \%$ level when testing the null hypothesis that $\mathrm{ICC}_{0}=0.5$ (power $=0.8$ ).

\section{DISCUSSION}

In the present study, n10 latency, peak-to-peak amplitude and AR for peak-to-peak amplitude were found to have excellent reliability in response to air conducted sound. The excellent reliability of these oVEMP parameters may be due to several factors. Firstly, the surface area of the cheek is smaller than that of the skin overlying the SCM, so there is less room for error in proper and optimal placement of the electrodes. Secondly, upgaze may be produce less fatigue in the muscle of interest than does flexing or rotating the neck, which may also lead to less intersession variation as well (35). Thirdly, there may be less variability in body habitus and soft tissue depth on the cheek than on the neck. Fourthly, the oVEMP response is an excitatory potential measured in the midst of relatively small background noise of extraocular muscle activation. In contrast, the cVEMP response is a small modulation in a relatively noisy background of SCM contraction (13). The former might be expected to be more repeatable a measure than the latter. The present study also found that the p15 latency demonstrated fair-to-good reliability. The discrepancy between n10 and P15 latency 
reliability values for the click stimulus may suggest that $\mathrm{n} 10$ has better clinical significance than 15 . Moreover, the p15 component has not been systematically examined and may even not be vestibular in origin. Therefore, n10 latency, peak-to-peak amplitude and AR for peak-to-peak amplitude may be more reliable parameters when measuring the oVEMP response.

In this study, we followed previous conventions for the classification of ICC values for test-retest reliability in VEMP testing [ie, ICC $\geq 0.75$ indicates excellent reliability and ICC $<0.40$ indicates poor reliability] $(10-12)$. However, one should keep in mind that these classifications are somewhat arbitrary. An ICC value is the ratio of the variance of a oVEMP parameter due to subject differences divided by the sum of variances due to subject differences and measurement errors. We chose to set 0.5 as a minimally meaningful ICC value because this is the point at which half of the variance in a measure between testing sessions can be ascribed to subject differences. A highly conservative assumption would be that the rest of the variance is due to "noise" that is not of clinical significance. However, test-retest reliability may actually be influenced by physiological variation in a healthy subject's response at different testing sessions, which may artificially deflate the ICC.

Nevertheless, we followed the conservative criterion and marked as "excellent" those reliability values in (Tables 2) which the ICC values were $>0.8$ and the $95 \%$ confidence intervals were $\geq 0.5$.

Cervical VEMP in response to air-conducted sound is well-established as measures of saccular function while ocular VEMP may test utricular function. We found that excellent overall oVEMP reliability for healthy pilots is comparable to that showed in the studies by Nguyen et al (14) and Maes et al (12). Therefore oVEMP is reliable to be a useful addition to the vestibular test battery in the medical selection and physiological assessment of fighter pilots. 
Meng et al

\section{CONCLUSION}

Ocular vestibular evoked myogenic potential can evaluate utricular and superior vestibular nerve function. In the study of 20 healthy pilots, good to excellent test-retest reliability has been established for overall parameters of oVEMP, with the latency of n10 more reliable than the latency of p15. It implies that oVEMP test is reliable in the vestibular evaluation of pilots. 


\section{oVEMP Reliability Pilots}

\section{REFERENCES}

1. Halmagyi GH, Aw ST, Cremer PD, Curthoys IS, Todd MJ. Impulsive testing of individual semicircular canal function. Ann NY Acad Sci 2001 ;942:192-200.

2. Brantberg K. Vestibular evoked myogenic potentials (VEMPs): usefulness in clinical neurotology. Semin Neurol $2009 ; 29$ : 541-47.

3. Hsu YS, Wang SJ, Young YH. Ocular vestibular-evoked myogenic potentials in children using air conducted sound stimulation. Clin Neurophysiol 2009; $120: 1381-85$.

4. Manzari L, Tedesco AR, Burgess AM, Curthoys IS. Ocular and cervical vestibular-evoked myogenic potentials to bone conducted vibration in Ménière's disease during quiescence vs during acute attacks. Clin Neurophysiol 2010; 121:1092-1101.

5. Murofushi T, Wakayama K, Chihara Y. oVEMP to air-conducted tones reflects functions of different vestibular populations from cVEMP? Eur Arch Otorhinolaryngol 2010;267: 995-96.

6. International Standardization Organization. Acoustics-Statistics distribution of hearing thresholds as a function of age. Geneva.2000; ISO 7029(2000).

7. Sandhu JS, George SR, Rea PA. The effect of electrode positioning on the ocular vestibular evoked myogenic potential to air-conducted sound. Clin Neurophysiol. 2013; 124: 1232-6.

8. Wang SJ, Jaw FS, Young YH. Optimizing the bandpass filter for acoustic stimuli in recording ocular vestibular-evoked myogenic potentials. Neurosci Lett 2013, 42:12-6. 
9. Wang SJ, Jaw FS, Young YH. Ocular vestibular evoked myogenic potentials elicited from monaural versus binaural acoustic stimulation. Clin Neurophysiol 2009; 120: 420-23.

9. Versino M, Colnaghi S, Callieco R, et al. Vestibular evoked myogenic potentials: test-retest reliability. Functional Neurology 2001; 16: 299-309.

10. Isaradisaikul S, Strong DA, Moushey JM, et al. Reliability of vestibular evoked myogenic potentials in healthy subjects. Otol Neurotol 2008; 29: 542-44.

11. Maes L, Vinck BM, De Vel E, et al. The vestibular evoked myogenic potential: a test-retest reliability study. Clin Neurophysiol 2009; 120: 594-600.

12. Fuchs AF, Binder MD. Fatigue resistance of human extraocular muscles. J Neurophysiol 1983; 49: 28-34.

14. Nguyen KD, Welgampola MS, Carey JP. Test-retest reliability and age-related characteristics of the ocular and cervical vestibular evoked myogenic potential test Otol Neurotol. 2010; 31: 793-802. 